\title{
Dental notation
}

The real problem with the Palmer notation is

that it appears to be extremely hard to convert it to HTML, the programming language of the Internet... As we move further into electronic publishing these difficulties becomes more relevant, urgent and important

Mike Grace m.grace.bdj@bda-dentistry.org.uk

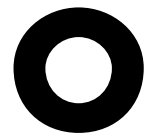

n page 268 of this issue we are publishing a new style for the way teeth are notated in the BDJ, and by coincidence on page 233 we are also publishing a letter describing how people can use the familiar Palmer style of tooth notation in two word-processing software packages. Although unrelated, the two items in this issue of the Journal suggest that there is a growing need for an internationallyagreed method of tooth notation that is easy to understand and easy to use with the increasing use of IT in the future.

As we all know, dental notation is the name for the way we identify teeth when we are writing them down. Thus the upper right central incisor (usually called the upper right one) will be written as 1 in the UK (Palmer method) and 11 in the FDI notation. The Palmer method is popular in the UK and easy to understand for those trained in the UK as it is based on a grid of teeth as if looking at the patient. It is also easy to write on the record card but not so easy to write on a typewriter, and even harder to write using a word processor (as detailed in the letter by David Lewis in this issue).

However the real problem with the Palmer notation is that it appears to be extremely hard to convert it to HTML, the programming language of the Internet. This means that the programmers converting the files we use at the $B D J$ to make up each issue on the $B D J$ web page have real problems. As we move further into electronic publishing these difficulties becomes more relevant, urgent and important.

The Editorial Board debated the subject of tooth notation a few years ago because of the pressure to convert to the FDI system of notation for the sake of international understanding. The final decision at that time was to continue using the Palmer notation because UK dentists still find the FDI notation confusing, and the $B D J$ is still the British Dental Journal. However, we have been using the FDI notation as well, placing the tooth number in brackets after the Palmer notation. Thus the upper left seven would be written as 17 (27).

Initially this system worked quite well, although occasionally confusion occurred and sometimes papers would only contain the Palmer notation or the FDI notation. Although we were aware it was not ideal we continued to follow this practice, partly because of the difficulty of deciding how to proceed, partly because of the sensitivities involved (people seem to get very attached to their ideas on tooth notation) and partly through natural lethargy.

We now believe the time has come to make a major change because of the increasing use of the web and the difficulties the Palmer system is causing. The simplest way forward (we currently believe) is to convert the Palmer system into a simple UR or LL shorthand (UR=upper right, $\mathrm{UL}=$ upper left, $\mathrm{LL}=$ lower left and $\mathrm{LR}=$ lower right). Thus we are still using the same system, but writing it in a different way to avoid the need for using a grid. Therefore $\angle 7$ (27) would be written UL7 (27). We still want to keep the FDI notation but feel it is premature to drop the Palmer system completely.

I am aware that there is some software to help write the Palmer notation as a grid, but we have had difficulties writing groups of teeth (such as $\left.\begin{array}{ll|l|l|}543 & 234 & 7\end{array}\right)$ and are not aware it is suitable for the web. Even if it was I personally cannot see a problem changing to the UR or LL system, except for the fact that visually the Palmer grid is quicker to understand at a glance.

Our decision has been taken after much deliberation. Doubtless there will be those who consider it a step backwards, and others who feel we have not moved far enough and perhaps should be changing to the FDI notation completely. The change is experimental, and we will have to wait to see how well it works. 